\title{
Characteristics and Antibiotic Resistance of Haemophilus influenzae in Children with Lower Respiratory Tract Infection in Chengdu, China
}

\author{
Qin Wang (iD ${ }^{1,}{ }^{*}$, Cheng-Gui Liu ${ }^{1}$, Jian Xu ${ }^{1}$, Qin Zhang ${ }^{1}$, Chong-Hui Zhao ${ }^{1}$ and Pei-Pei Song ${ }^{1}$ \\ ${ }^{1}$ Department of Clinical Laboratory, Chengdu Women's and Children's Central Hospital, School of Medicine, University of Electronic Science and Technology of China, \\ Chengdu 611731, China \\ "Corresponding author: Department of Clinical Laboratory, Chengdu Women's and Children's Central Hospital, School of Medicine, University of Electronic Science and \\ Technology of China, Chengdu 611731, China. Email: cqwq1986925@sina.com
}

Received 2021 March 22; Revised 2021 May 02; Accepted 2021 May 04.

\begin{abstract}
Background: Haemophilus influenzae is an opportunistic pathogen of the human respiratory tract. Haemophilus influenzae can cause not only respiratory tract infection in children but also otitis media, epiglottitis and sinusitis. With the widespread use of antibiotics, the positive rate of $\beta$-lactamase in $H$. influenzae is increasing, and the rate of antimicrobial resistance is also increasing, which increases the difficulty of clinical treatment.

Objectives: To study the infection characteristics of patients and the antibiotic resistance of $H$. influenzae in lower respiratory tract samples of children in Chengdu, so as to provide a reference for its clinical diagnosis and the rational use of antibiotics.

Methods: Sputum samples of 15891 children aged 0-14 years with lower respiratory tract infection were collected. Haemophilus influenzae was cultured and identified, its drug susceptibility tested, and the results determined according to the guidelines of CLSI 2020.

Results: A total of 15891 clinical isolate strains in sputum were detected for drug sensitivity from December 2018 to January 2020 , of which 5488 were H. influenzae, accounting for $34.54 \%$ (5488/15891). The sex of children infected with H. influenzae was not skewed (P $>0.05$ ). The detection rate of $H$. influenzae was the highest in children aged $7-11$ months, and the lowest was in the age group $\leq 28 \mathrm{~d}$. The detection rate was the highest in spring and the lowest in autumn. The positive rate of $\beta$-lactamase was $92.0 \%$, the resistance rate to ampicillin was $92.0 \%$, the sensitivity to amoxicillin/clavulanate was $70.2 \%$, and the sensitivity to cefotaxime, ofloxacin, tetracycline, chloramphenicol, and rifampicin was more than $90.0 \%$.

Conclusions: Children aged 7 months to 14 years were generally susceptible to $H$. influenzae in spring, and the positive rate of $\beta$ lactamase was high. Doctors should refer to the infection characteristics and drug resistance of $H$. influenzae and choose antibiotics correctly to better control the infection.
\end{abstract}

Keywords: Children, Haemophilus influenzae, Antibiotic resistance, Chengdu, China

\section{Background}

Haemophilus influenzae is a Gram-negative bacillus that has a capsule and a high colonization rate in the respiratory tract and can cause infection in many parts of the body, including otitis media, epiglottis, sinusitis, and pneumonia (1). Children are generally susceptible to bacteria because of their special physiological and immune status. The main route of transmission of $H$. influenzae is respiratory droplets, and a special route of transmission in children is through inhalation of maternal amniotic fluid or exposure to genital tract secretions during spontaneous delivery (2). Due to the strong pathogenicity of $H$. influen$z a e$, if children are not actively treated after infection, it may lead to serious consequences, such as bacteremia and meningitis (3). With the overuse of antibiotics and the de- velopment of various drug resistance mechanisms, the resistance rates of $H$. influenzae to ampicillin and first-line cephalosporins are rising $(4,5)$. In recent years, due to the irregular use of antibiotics in children, the drug resistance rate of $H$. influenzae has increased (6). Laboratory support is urgently needed for its treatment, including early identification of H. influenzae and selection of antibiotics it will be sensitive to (7).

\section{Objectives}

The aim of this study was to investigate $H$. influen$z a e$ infections in the lower respiratory tract of children in Chengdu and to evaluate the antibiotic resistance in the Chengdu area between February 2018 and January 2020, so 
as to provide a reference for the clinical diagnosis of $\mathrm{H}$. influenzae infection and the rational use of antibiotics.

\section{Methods}

\subsection{Subjects}

A total of 15891 clinical isolate strains in sputum were collected from outpatients and inpatients at Chengdu Women's and Children's Central Hospital, School of Medicine, University of Electronic Science and Technology of China. Patients from the Chengdu region were selected over a 2-year period from February 2018 to January 2020. The subjects were between 1 day and 14 years of age and included 9236 males and 6655 females. There were 1786 newborns ( $\leq 28$ days), 5118 infants (29 days- 6 months) and 2058 babies aged 7 months to 11 months; 6010 children were aged 1-3 years, 792 were aged 4 - 6 years, and 127 were aged 7 - 14 years. According to the season, the sputum samples were divided into four groups: spring, summer, autumn and winter, including 3987 cases in spring, 3267 cases in summer, 3266 cases in autumn and 5371 cases in winter.

\subsection{Specimen Collection}

According to the literature (8), specimens were collected by physicians and put into a sterile container. Then the specimens were sent to the laboratory, and proper screening of sputum with Gram staining was performed immediately. A total of 20 - 40 visual fields were detected under microscope, according to the qualified standard of sputum: sputum specimens containing $>25$ leukocytes but $<10$ squamous epithelial cells per low-power field (LPF, $\times 100$ ) were considered of good quality for bacterial culture in patients. Otherwise, the sputum sample was considered contaminated by saliva and rejected ( 9 ).

\subsection{Identification of Haemophilus influenzae}

Sputum specimens were cultured in accordance with the operational requirements of the fourth edition of the National Rules for Clinical Examination. The plates were placed at $35^{\circ} \mathrm{C}$ and cultured in a $5-10 \% \mathrm{CO}_{2}$ incubator for 18 - 24 hours. Clear or translucent, flat, moist, dewdrop colonies were selected, confirmed as Gram-negative bacilli under the microscope, and then identified using the VITEK 2 compact automatic microbial identification system (BioMerieux, France) (7).

\subsection{Antimicrobial Susceptibility Testing}

According to the Clinical and Laboratory Standards Institute (CLSI) disk diffusion methods, the minimum inhibitory concentrations (MICs) of ampicillin, amoxicillin-clavulanic acid, cefuroxime, cefaclor, cefotaxime, tetracycline, ofloxacin, chloramphenicol, trimethoprim-sulfamethoxazole, and rifampicin were determined by an ATB HAEMO drug sensitivity testing system(BioMerieux, France) using Haemophilus test medium (HTM) (Chongqing PangTong Medical Devices Co., China). The interpretative breakpoints were based on the CLSI criteria (http://www.clsi.org/clinical breakpoints/). Testing was performed at $35^{\circ} \mathrm{C}$ for 18 - 24 hours. ATCC reference H. influenzae 49247 (Microbiologics, USA) was used for quality control. $\beta$-Lactamase production was revealed by nitrocefin sticks. The antibiotic susceptibility results were determined according to the CLSI 2020 standard and were analyzed by WHONET 5.6 statistical software $(10,11)$.

\subsection{Statistical Analysis}

SPSS Statistics version 22 (SPSS Inc. Chicago, IL, USA) was used to analyze the data. The statistical test used for all data was the chi-square test. $\mathrm{P}<0.05$ was considered to be statistically significant.

\section{Results}

\subsection{Detection Rate of Haemophilus influenzae}

A total of 5488 strains of $H$. influenzae were detected from 15891 clinical isolate strains in sputum collected from February 2018 to January 2020, for a positive rate of $34.54 \%$.

4.2. Relationship Between Haemophilus influenzae Infection and Sex

Among the 5488 strains of $H$. influenzae detected from 15891 clinical isolate strains in sputum, there were 3197 males and 2291 females. Sex comparison showed that there was no significant difference $(\mathrm{P}>0.05)$, as shown in Table 1.

\subsection{Detection Rate of Haemophilus influenzae in Patients in Dif- ferent Age Groups}

Patients were divided into six groups: $(1) \leq 28$ days old; (2) 29 days to 6 months old; (3) 7 to 11 months old; (4) 1 to 3 years old; (5) 4 to 6 years old; and (6) 7 to 14 years old. The results showed that the detection rates of $H$. influenzae in groups 3, 4, 5 and 6 were higher than those in groups 1 and 2. Group 3 had the highest detection rate, and group 1 had the lowest infection rate (Table 2 ). 


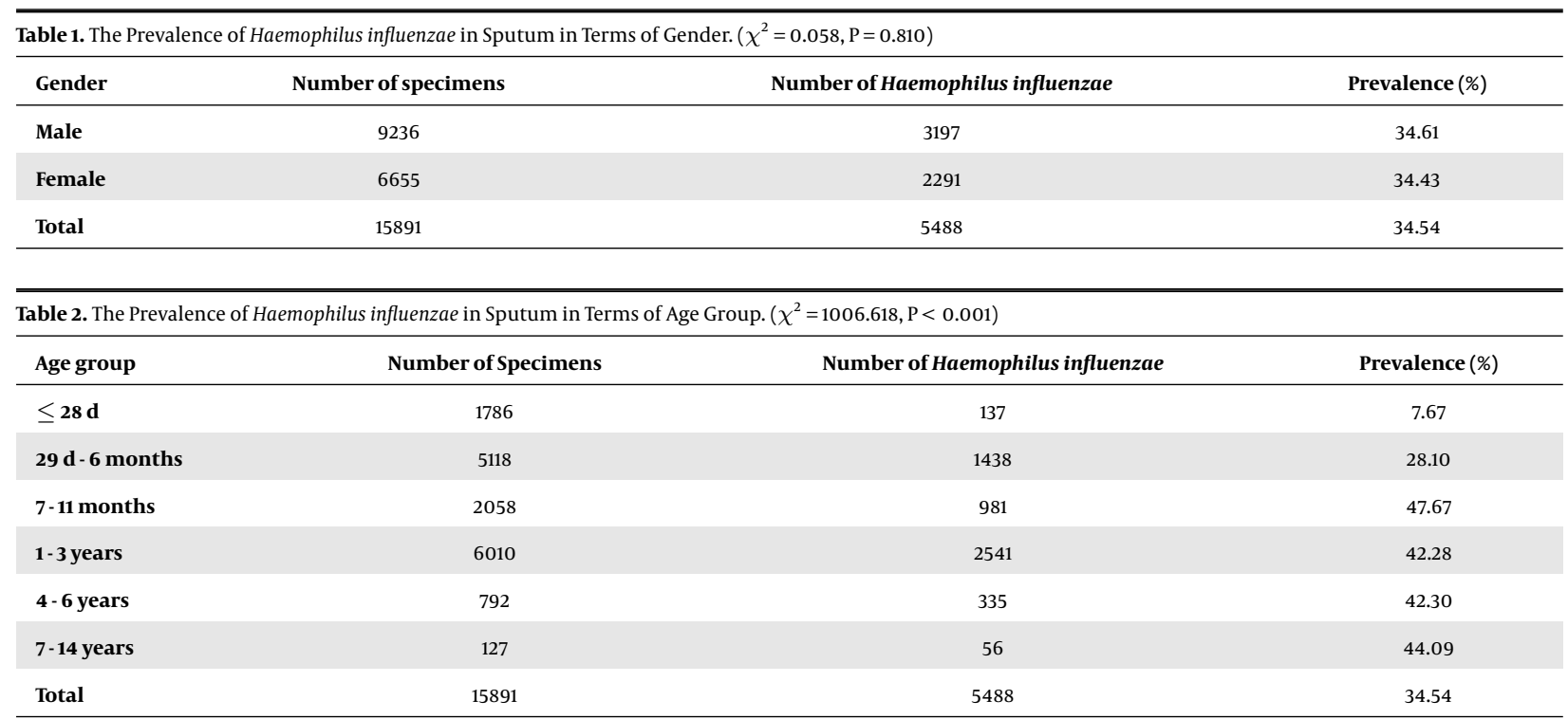

4.4. Relationship Between Haemophilus influenzae Infection and Season

Among the 5488 strains of $H$. influenzae detected, the detection rate in children in summer vs. winter was not significantly different, but it was significantly different between spring and autumn. The prevalence of H. influenzae in sputum was the highest in spring and the lowest in autumn, as shown in Table 3.

\subsection{Analysis of Antimicrobial Susceptibility Testing}

Of the 5488 strains detected, 5049 (92.0\%) were positive for $\beta$-lactamase. The resistance rate of the $H$. influen$z a e$ isolates to ampicillin was $92.0 \%$. The resistance rates to cefuroxime, cefaclor and trimethoprim-sulfamethoxazole were all more than $60.0 \%$. It was found that the sensitive rate of $H$. influenzae to tetracycline, chloramphenicol and rifampicin was more than $90.00 \%$. The sensitive rate to amoxicillin/clavulanate was $70.2 \%$. No resistance to cefotaxime or ofloxacin was detected (Table 4).

\section{Discussion}

Haemophilus influenzae is a common pathogen of community-acquired infection in children $(12,13)$. Bacteria mainly cause infection in children, including pneumonia, otitis media, cellulitis, arthritis, meningitis, bloodstream infection and other diseases, making it a serious threat to children's health $(14,15)$. In recent years, with the overuse of antibiotics in children, the drug-resistant strains of $H$. influenzae are growing in number, which brings great difficulties to treatment (7).
Owing to the imperfect development of children's respiratory system, relatively low immune function and the high microbial colonization rate, the infection rate of $H$. influenzae in children has been very high $(16,17)$. For economic reasons and lack of awareness of $H$. influenzae, although the Chinese government recommended that infants be vaccinated against $H$. influenzae nationwide and launched a self-pay H. influenzae type b strain vaccine, there are still many unvaccinated children, and the infection caused by non-type b or non-typeable H. influenzae strains is still a serious problem. These strains are currently the most common cause of invasive $H$. influenzae infection in many countries $(18,19)$.

In this study, we conducted a retrospective investigation to study the epidemic characteristics and antibiotic resistance of $H$. influenzae in children aged $0-14$ years with lower respiratory tract infection in Chengdu, China. Our results showed that 5488 strains of $H$. influenzae were detected, and the total positive rate of $H$. influenzae in this study was $34.54 \%$. This indicated that $H$. influenzae infection was high in children with lower respiratory tract infections in the Chengdu region. This result supports one previous investigation that suggested that $H$. influenza was one of the most common causes of community-acquired pneumonia in children (20).

In our study, sex comparison showed that there was no significant difference. This result indicates that there was no sex difference in $H$. influenzae infection in children in Chengdu. The age distribution of $H$. influenzae infection in our hospital was most concentrated from 29 days to 14 years old, as the detection rates of H. influenzae in age groups 3, 4, 5 and 6 were significantly higher than those 


\begin{tabular}{|c|c|c|c|c|}
\hline \multirow{2}{*}{$\begin{array}{l}\text { Seasons } \\
\text { Spring }\end{array}$} & Number of Specimens & \multicolumn{2}{|c|}{ Number of Haemophilus influenzae } & \multirow{2}{*}{$\begin{array}{c}\text { Prevalence (\%) } \\
50.67\end{array}$} \\
\hline & 3987 & & & \\
\hline Summer & 3267 & & & 33.03 \\
\hline Autumn & 3266 & & & 18.52 \\
\hline Winter & 5371 & & & 33.22 \\
\hline Total & 15891 & & & 34.54 \\
\hline Antibiotic & $\mathbf{n}$ & $S$ & I & $\mathbf{R}$ \\
\hline Ampicillin & 5488 & $439(8.0)$ & $0(0.0)$ & $5049(92.0)$ \\
\hline Amoxicillin/clavulanate & 5488 & $3853(70.2)$ & $0(0.0)$ & $1635(29.8)$ \\
\hline Cefuroxime & 5488 & $1613(29.4)$ & $209(3.8)$ & $3666(66.8)$ \\
\hline Cefaclor & 5488 & $1164(21.2)$ & $263(4.8)$ & $4061(74.0)$ \\
\hline Cefotaxime & 5488 & $5488(100.0)$ & $0(0.0)$ & $0(0.0)$ \\
\hline Tetracycline & 5488 & $5241(95.5)$ & $11(0.2)$ & $236(4.3)$ \\
\hline Ofloxacin & 5488 & $5488(100.0)$ & $0(0.0)$ & $0(0.0)$ \\
\hline Chloramphenicol & 5488 & $5263(95.9)$ & $5(0.1)$ & $220(4.0)$ \\
\hline $\begin{array}{l}\text { Trimethoprim- } \\
\text { sulfamethoxazole }\end{array}$ & 5488 & $1690(30.8)$ & $0(0.0)$ & $3798(69.2)$ \\
\hline Rifampicin & 5488 & $5483(99.9)$ & $0(0.0)$ & $5(0.1)$ \\
\hline
\end{tabular}

Abbreviations: S, susceptible; I, intermediate; R, resistant.

${ }^{a}$ Values are expressed as No. (\%)

in age groups 1 and $2(\mathrm{P}<0.05)$. Our study also showed that the detection rates of $H$. influenzae in the 7 -11-month age group were the highest, and newborns under 28 days had the lowest infection rate. The reason for this result may be related to their pulmonary function and immunity. Since most of the patients came from the community, children less than 6 months old had maternal antibodies and higher resistance to community-acquired pneumonia. Several studies have shown that $H$. influenzae infection shows an obvious seasonal distribution, usually occurring in winter and spring, or between February and May $(7,20)$. Our study showed that the detection rates of $H$. influenzae in sputum were the highest in spring and the lowest in autumn, which was not consistent with the previous studies. This indicated that the seasonal distribution of $H$. influenzae infection may be different due to changes in climatic conditions.

With the development of various drug resistance mechanisms, such as $\beta$-lactamase activity and modified bacterial penicillin binding protein (21), the range of antibiotics active against $H$. influenzae has gotten narrower, especially given the limited set of antibiotics that can be given to children. It is of great significance to study the antibiotic resistance of $H$. influenzae in children. Most $H$. influenzae strains are phenotypically $\beta$-lactamase negative and ampicillin susceptible (BLNAS), so ampicillin and amoxicillin/clavulanate, as well as third-generation cephalosporins such as cefotaxime, are among the drugs of choice for the treatment of H. influenzae infections (22), but in our study, of the 5488 strains detected, 5049(92.0\%) were positive for $\beta$-lactamase. The $H$. influenzae ampicillin resistance rate was $92.0 \%$, which was higher than what had been found in previous studies in China (58.1\%) (7), Iran (54.8\%), Germany $(11.6 \%)$ and Poland $(29.1 \%)(7,23)$, but close to the ampicillin resistance rate in South Korea (69.4\%) (7).

These data suggested that the overall enzyme production rate of $H$. influenzae was high and had an increasing trend, which was also the main reason for the drug resistance of $H$. influenzae. The differences in the above results may also be due to differences in geographical location or the use of antibiotics by children. The sensitive rate to amoxicillin/clavulanate was $70.2 \%$. Therefore, single penicillin-type drugs, such as ampicillin and amoxicillin, are not recommended in clinical treatment, but $\beta$ lactamase inhibitors are recommended for antibacterial treatment. The resistance rate of cefotaxime was very low, 
which was consistent with previous studies in Taiwan and Germany $(3,22)$. The resistance rates to cefuroxime, cefaclor and trimethoprim-sulfamethoxazole were all more than $60.0 \%$, and the sensitive rates of H. influenzae to tetracycline, chloramphenicol and rifampicin were more than 90.0\%. Due to the limitations of their use in children, we do not recommend ofloxacin, tetracycline, and chloramphenicol for children. Since $H$. influenzae showed low resistance rates to amoxicillin/clavulanate and cefotaxime, they have good antibacterial activity in children with $H$. influenzae infection and might be appropriate for this population.

In recent years, the resistance rate of $H$. influenzae to ampicillin has gradually increased. Microbiological laboratories should pay attention to and strengthen the culture and drug resistance surveillance of $H$. influenzae. At the same time, physicians should choose antibiotics reasonably according to the results of drug sensitivity tests, control the spread and prevalence of drug-resistant bacteria, and avoid unreasonable drug use.

\subsection{Conclusions}

In conclusion, children aged 7 months to 14 years were generally susceptible to $H$. influenzae in spring, and the positive rate of $\beta$-lactamase reached 92.0\%. Amoxicillin/clavulanate or cefotaxime can be used as the first choice for treatment. To better control the infection, physicians can refer to these characteristics and antibiotic resistance of $H$. influenzae for diagnosis and treatment.

\section{Acknowledgments}

We are thankful for the funds given to support the experiments and to all coauthors for devoting their time to the manuscript.

\section{Footnotes}

Authors' Contribution: Qin Wang conducted the specimen studies and drafted the manuscript; Cheng-Gui Liu contributed to the study design; Jian Xu, Qin Zhang and Pei-Pei Song participated in the susceptibility testing; Chong-Hui Zhao contributed to the statistical analysis.

Conflict of Interests: There were no conflicts of interest for any of the authors.

Ethical Approval: This study was done in accordance with the Helsinki Declaration and was approved by the Medical Ethics Committee at Chengdu Women's and Children's Central Hospital, Chengdu, China ((2013)2, Medical Ethics Committee, CWCCH). Consent from each participant was waived because the present study was retrospective. Because all patient data were analyzed anonymously, no additional informed consent was required.

Funding/Support: This study was supported by the Sichuan Provincial Science and Technology Department Research Foundation of China (2013FZ0080), the Chengdu Science and Technology Bureau Research Foundation of China (2015-HM0100623-SF) and the Science and Technology Project of the Health Planning Committee of Sichuan Province (16PJ076).

\section{References}

1. Tsang RSW, Ulanova M. The changing epidemiology of invasive Haemophilus influenzae disease: Emergence and global presence of serotype a strains that may require a new vaccine for control. Vaccine. 2017;35(33):4270-5. doi: 10.1016/j.vaccine.2017.06.001. [PubMed: 28666758].

2. Cox RA, Slack MP. Clinical and microbiological features of Haemophilus influenzae vulvovaginitis in young girls. J Clin Pathol. 2002;55(12):961-4. doi: 10.1136/jcp.55.12.961. [PubMed: 12461068]. [PubMed Central: PMC1769841].

3. Su PY, Huang AH, Lai CH, Lin HF, Lin TM, Ho CH. Extensively drug-resistant Haemophilus influenzae - emergence, epidemiology, risk factors, and regimen. BMC Microbiol. 2020;20(1):102. doi: 10.1186/s12866-020-01785-9. [PubMed: 32345232]. [PubMed Central: PMC7189504].

4. Nathwani D, Wood MJ. Penicillins. A current review of their clinical pharmacology and therapeutic use. Drugs. 1993;45(6):866-94. doi: 10.2165/00003495-199345060-00002. [PubMed: 7691496].

5. Low DE. Resistance trends in Haemophilus influenzae (PROTEKT years 1-3 [1999-20021). J Chemother. 2004;16 Suppl 6:49-61. doi: 10.1080/1120009x.2004.11782402. [PubMed: 15690685].

6. Magiorakos AP, Srinivasan A, Carey RB, Carmeli Y, Falagas ME, Giske CG, et al. Multidrug-resistant, extensively drug-resistant and pandrug-resistant bacteria: an international expert proposal for interim standard definitions for acquired resistance. Clin Microbiol Infect. 2012;18(3):268-81. doi: 10.1111/j.1469-0691.2011.03570.x. [PubMed: 21793988].

7. Wang HJ, Wang CQ, Hua CZ, Yu H, Zhang T, Zhang H, et al. Antibiotic resistance profiles of Haemophilus influenzae isolates from children in 2016: A multicenter study in China. Can J Infect Dis Med Microbiol. 2019;2019:6456321. doi: 10.1155/2019/6456321. [PubMed: 31485283]. [PubMed Central: PMC6710757].

8. Joyce SM. Sputum analysis and culture. Ann Emerg Med.1986;15(3):3258. doi: 10.1016/s0196-0644(86)80576-5. [PubMed: 3946881].

9. Roson B, Carratala J, Verdaguer R, Dorca J, Manresa F, Gudiol F. Prospective study of the usefulness of sputum Gram stain in the initial approach to community-acquired pneumonia requiring hospitalization. Clin Infect Dis. 2000;31(4):869-74. doi: 10.1086/318151. [PubMed: 11049763].

10. Ranjbar R, Farsani FY, Dehkordi FS. Phenotypic analysis of antibiotic resistance and genotypic study of the vacA, cagA, iceA, oipA and babA genotypes of the Helicobacter pylori strains isolated from raw milk. Antimicrob Resist Infect Control. 2018;7:115. doi:10.1186/s13756-018-0409y. [PubMed: 30288255]. [PubMed Central: PMC6162967].

11. Abdolmaleki Z, Mashak Z, Safarpoor Dehkordi F. Phenotypic and genotypic characterization of antibiotic resistance in the methicillinresistant Staphylococcus aureus strains isolated from hospital cockroaches. Antimicrob Resist Infect Control. 2019;8:54. doi: 10.1186/s13756019-0505-7. [PubMed: 30911380]. [PubMed Central: PMC6416839]. 
12. Giufre M, Fabiani M, Cardines R, Riccardo F, Caporali MG, D'Ancona F, et al. Increasing trend in invasive non-typeable Haemophilus influenzae disease and molecular characterization of the isolates, Italy, 20122016. Vaccine. 2018;36(45):6615-22. doi: 10.1016/j.vaccine.2018.09.060. [PubMed: 30292458].

13. Nagai K, Kimura O, Domon H, Maekawa T, Yonezawa D, Terao Y. Antimicrobial susceptibility of Streptococcus pneumoniae, Haemophilus influenzae, and Moraxella catarrhalis clinical isolates from children with acute otitis media in Japan from 2014 to 2017. Infect Chemother. 2019;25(3):229-32. doi:10.1016/j.jiac.2018.08.018. [PubMed: 30279114].

14. Kaur R, Morris M, Pichichero ME. Epidemiology of acute otitis media in the postpneumococcal conjugate vaccine era. Pediatrics. 2017;140(3). doi: 10.1542/peds.2017-0181. [PubMed: 28784702]. [PubMed Central: PMC5574724 conflicts of interest to disclose].

15. Tsang RSW, Shuel M, Whyte K, Hoang L, Tyrrell G, Horsman G, et al. Antibiotic susceptibility and molecular analysis of invasive Haemophilus influenzae in Canada, 2007 to 2014. J Antimicrob Chemother. 2017;72(5):1314-9. doi: 10.1093/jac/dkw565. [PubMed: 28137937]. [PubMed Central: PMC5890693].

16. Giufre M, Daprai L, Cardines R, Bernaschi P, Rava L, Accogli M, et al. Carriage of Haemophilus influenzae in the oropharynx of young children and molecular epidemiology of the isolates after fifteen years of H. influenzae type b vaccination in Italy. Vaccine. 2015;33(46):6227-34. doi: 10.1016/j.vaccine.2015.09.082. [PubMed: 26440924].

17. Monge S, Mollema L, de Melker H, Sanders E, van der Ende A, Knol M. Clinical characterization of invasive disease caused by Haemophilus influenzae serotype $\mathrm{b}$ in a high vaccination coverage setting. J Pedi- atric Infect Dis Soc. 2019;8(3):261-4. doi:10.1093/jpids/piy020. [PubMed: 29579288].

18. Van Eldere J, Slack MP, Ladhani S, Cripps AW. Non-typeable Haemophilus influenzae, an under-recognised pathogen. Lancet Infect Dis. 2014;14(12):1281-92. doi: 10.1016/S1473-3099(14)70734-0. [PubMed: 25012226].

19. Slack MPE. The evidence for non-typeable Haemophilus influenzae as a causative agent of childhood pneumonia. Pneumonia (Nathan). 2017;9:9. doi: 10.1186/s41479-017-0033-2. [PubMed: 28702311]. [PubMed Central: PMC5483294].

20. Li JP, Hua CZ, Sun LY, Wang HJ, Chen ZM, Shang SQ. Epidemiological features and antibiotic resistance patterns of Haemophilus in fluenzae originating from respiratory tract and vaginal specimens in pediatric patients. J Pediatr Adolesc Gynecol. 2017;30(6):626-31. doi: 10.1016/j.jpag.2017.06.002. [PubMed: 28629795].

21. Neu HC. The crisis in antibiotic resistance. Science. 1992;257(5073):1064-73. doi: 10.1126/science.257.5073.1064. [PubMed: 1509257].

22. Lam TT, Nurnberg S, Claus H, Vogel U. Molecular epidemiology of imipenem resistance in invasive Haemophilus influenzae infections in Germany in 2016. J Antimicrob Chemother. 2020;75(8):2076-86. doi: 10.1093/jac/dkaa159. [PubMed: 32449913]

23. Vaez H, Sahebkar A, Pourfarzi F, Yousefi-Avarvand A, Khademi F Prevalence of antibiotic resistance of Haemophilus influenzae in iran- a meta-analysis. Iran J Otorhinolaryngol. 2019;31(107):349-57. doi: 10.22038/ijorl.2019.34363.2137. [PubMed: 31857979]. [PubMed Central: PMC6914328]. 